\title{
Les statuts constitutionnels des peuples finno-ougriens dans la Fédération de Russie
}

The Constitutional Status of the Finno-Ugric Peoples in the Russian Federation КОНСТИТУЦИОННО-ПРАВОВЫЕ СТАТУСЫ ФИННО-УГОРСКИХ НАРОДОВ В РОССИЙСКОЙ ФЕДЕРАЦИИ

\section{Vladimir Krjažkov}

Traducteur : Franck Léonard

\section{OpenEdition}

\section{Journals}

Édition électronique

URL : https://journals.openedition.org/efo/7105

DOI : 10.4000/efo.7105

ISSN : 2275-1947

\section{Éditeur}

INALCO

\section{Édition imprimée}

ISBN : 978-2-343-12463-6

ISSN : 0071-2051

\section{Référence électronique}

Vladimir Krjažkov, « Les statuts constitutionnels des peuples finno-ougriens dans la Fédération de Russie », Études finno-ougriennes [En ligne], 48| 2017, mis en ligne le 18 juillet 2017, consulté le 08 juillet 2021. URL : http://journals.openedition.org/efo/7105 ; DOI : https://doi.org/10.4000/efo.7105

Ce document a été généré automatiquement le 8 juillet 2021.

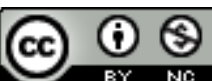

Études finno-ougriennes est mis à disposition selon les termes de la Licence Creative Commons Attribution - Pas d'Utilisation Commerciale 4.0 International. 


\section{Les statuts constitutionnels des peuples finno-ougriens dans la Fédération de Russie}

The Constitutional Status of the Finno-Ugric Peoples in the Russian Federation КОНСТИТУЦИОННО-ПРАВОВЫЕ СТАТУСЫ ФИННО-УГОРСКИХ НАРОДОВ В

РОССИЙСКОЙ ФЕДЕРАЦИИ

Vladimir Krjažkov

Traduction : Franck Léonard

\section{NOTE DE L'AUTEUR}

La base « Consultant Plus » a été utilisée pour la préparation de cet article.

\section{Types et fondements des statuts constitutionnels des peuples finno-ougriens}

1 Les peuples finno-ougriens constituent un groupe particulier de peuples issus d'une même famille linguistique, qui se singularisent à l'intérieur de la population multinationale de la Fédération de Russie par la langue ${ }^{1}$. Tout en partageant quelques caractéristiques culturelles et linguistiques communes, ils se différencient par leurs statuts constitutionnels. En particulier, on peut distinguer :

- les peuples qui ont donné leurs noms aux Républiques éponymes au sein de la Fédération de Russie (art. 65 de la Constitution de la Fédération de Russie). C'est le cas des Mordves, des Oudmourtes, des Maris, des Komis et des Caréliens. Ils forment, en quelque sorte, les peuples constituants des sujets de la Fédération de Russie ${ }^{2}$. Les problèmes qui les concernent sont réglés directement par les organes du pouvoir des Républiques ; 
- les peuples qui sont qualifiés de " petits $^{3}$ » peuples autochtones. La Fédération de Russie garantit leurs droits conformément aux principes universellement reconnus, aux règles du droit international et aux conventions internationales de la Fédération de Russie (art. 69 de la Constitution de la Fédération de Russie). Une liste de ces peuples a été approuvée par le gouvernement de la Fédération de Russie (décret du 24 mars 2000, $\mathrm{n}^{\circ} 255^{4}$ ). Parmi ces peuples, les Finno-Ougriens sont : les Besermans, les Ingriens, les Votes, les Setos ainsi que les Khantys, les Mansis, les Vepses et les Sames, qui sont également inclus dans la liste des «petits» peuples autochtones du Nord, de Sibérie et d'Extrême-Orient (approuvée par une ordonnance du gouvernement de la Fédération de Russie du 17 avril 2006, n ${ }^{\circ}$ 536- ${ }^{5}$ ). Les droits et les garanties spécifiques de ces peuples sont déterminés par la loi fédérale du 30 avril 1999, "Sur les garanties des droits des peuples autochtones minoritaires de la Fédération de Russie $»^{6}$;

- les peuples qui constituent une minorité nationale (Finnois, Estoniens ${ }^{7}$, etc.). La Constitution de la Fédération de Russie garantit les droits de ces minorités (art.71, par. 1, c ; art. 72, par. $1, b)$.

2 Ainsi, la Constitution de la Fédération de Russie admet la diversité des statuts ou l'utilisation de structures étatiques différentes pour la résolution des problèmes des peuples finno-ougriens. Cette situation se trouve parfaitement justifiée sur le plan historique et correspond aux situations réelles de ces peuples ainsi qu'à leurs besoins.

Par ailleurs, la Constitution de la Fédération de Russie, en se fondant sur l'égalité juridique et sur l'autodétermination des peuples au sein de la Fédération de Russie (art. 5, par. 3), reconnaît que la terre et les autres ressources naturelles sont exploitées et protégées comme fondement de la vie et de l'activité des peuples qui vivent sur les territoires concernés (art.9, par. 1). Tous les peuples se voient garantir le droit à la conservation de leur langue maternelle et à la création des conditions pour son enseignement et son développement (art.68, par. 3). Conformément à la Constitution, le gouvernement garantit l'égalité des droits et des libertés individuelles indépendamment de la nationalité et de la langue (art. 19, par. 2), le droit pour chacun de déterminer et d'indiquer ou de ne pas déterminer et de ne pas indiquer son appartenance nationale (art.26, par.1), d'utiliser sa langue maternelle, de choisir librement une langue de communication, d'éducation, d'enseignement et de création artistique (art. 26, par. 2).

Il est également important de noter que la Constitution de la Fédération de Russie (art. 72) accorde à la Fédération de Russie et à ses sujets (les républiques, les oblast', les kraj, les okrug autonomes) une compétence partagée pour la protection des droits et des libertés individuelles, des droits des minorités nationales, de l'habitat séculaire et du mode de vie traditionnel des communautés ethniques minoritaires, leur protection sociale, les questions d'exploitation des ressources naturelles, de protection de l'environnement, des monuments historiques et culturels, d'éducation, les questions scientifiques et culturelles. Cela signifie que :

- le règlement de ces questions relève à la fois de la compétence de la Fédération de Russie et des sujets de celle-ci, qui sont solidairement responsables de l'état du droit dans ces domaines ;

- la législation fédérale laisse aux sujets de la Fédération de Russie une compétence pour la réglementation de situations juridiques particulières (décision de la Cour constitutionnelle de la Fédération de Russie du 15 novembre 2012, nº 26-p $\mathrm{p}^{8}$; 
- pour les matières mentionnées, et en vertu des principes généraux, d'abord la Fédération adopte les lois fédérales; puis sont adoptés les lois et les autres actes juridiques des sujets de la Fédération de Russie, conformément aux lois fédérales. Les actes juridiques régionaux ne peuvent pas contredire les lois fédérales et, en présence d'une telle contradiction, c'est la loi fédérale qui s'applique (art. 76, par. 2, 5 de la Constitution). La loi fédérale du 6 octobre 1999 «Sur les principes généraux d'organisation des organes législatifs (représentatifs) et exécutifs du pouvoir d'État des sujets de la Fédération de Russie ${ }^{9}$, qui met en œuvre les dispositions ci-dessus, admet que les sujets de la Fédération de Russie ont le droit d'élaborer une réglementation juridique particulière pour les matières relevant de la compétence partagée jusqu'à l'adoption de lois fédérales (art. 3, par. 2). Ils ont le droit d'élaborer non seulement des lois et d'autres actes juridiques réglementaires, mais également des programmes régionaux (art. 26.3, par. 3.1.). Il s'agit de :

- la loi de la Fédération de Russie du 25 octobre 1991, «Sur les langues des peuples de la Fédération de Russie ${ }^{10}$;

- la loi de la Fédération de Russie du 9 octobre 1992, «Fondements de la législation de la Fédération de Russie en matière culturelle $"^{11}$;

- la loi fédérale du 6 janvier 1999, «À propos de l'artisanat populaire " $^{12}$;

- la loi fédérale du 25 juin 2002, «Sur les objets du patrimoine culturel (monuments historiques et culturels) des peuples de la Fédération de Russie $»^{13}$;

- la loi fédérale du 29 décembre 2012, «À propos de l'enseignement dans la Fédération de Russie $»^{14}$.

7 La Constitution de la Fédération de Russie et les autres lois fédérales disposent d'une primauté et d'un effet direct sur tout le territoire de la Fédération (art. 4, par. 2 ; art. 15, par. 1 ; art. 76, par. 1 de la Constitution de la Fédération de Russie).

\section{Le statut constitutionnel des peuples finno-ougriens qui ont donné leurs noms aux Républiques éponymes}

8 Conformément à la Constitution de la Fédération de Russie, les Républiques sont juridiquement égales entre elles et vis-à-vis des autres sujets de la Fédération, notamment dans leurs relations avec les organes fédéraux du pouvoir d'État (art. 5, par. 1, 4). Elles disposent de leur Constitution et de leur législation (art. 5, par. 2), ont le droit de déterminer leurs propres langues officielles (art. 68, par. 2), disposent de deux représentants au Conseil de la Fédération, qui est une Chambre du Parlement de la Fédération de Russie (art. 85, par. 2), disposent, par le biais de l'organe législatif de la République, du droit d'initiative législative à la Douma d'État, qui est une Chambre de l'Assemblée fédérale (art. 104). Elles peuvent, par l'intermédiaire de leurs organes du pouvoir d'État, saisir la Cour constitutionnelle de la Fédération de Russie en vue de vérifier la conformité des lois fédérales, et des autres actes juridiques, à la Constitution de la Fédération de Russie (art. 125).

9 La protection légale des droits des peuples finno-ougriens est assurée directement dans les Républiques.

Études finno-ougriennes, 48 | 2017 
10 La République de Carélie. La population totale est de 643000 habitants, dont 507000 Russes $(78,8 \%)$ et 45570 Caréliens (7\%). Par ailleurs, $70 \%$ des Caréliens de toute la Fédération de Russie vivent dans la République ${ }^{15}$.

11 La Constitution de la République reconnaît que le nom «Karjala » est équivalent de celui de « République de Carélie » ou de «Carélie », que les particularités historiques et nationales de la République sont déterminées par l'habitation des Caréliens sur son territoire $\left(\right.$ art. $\left.1^{\mathrm{er}}\right)$. Les peuples de Carélie se voient garantir le droit à la préservation de leur langue natale ainsi que la création des conditions propices à son étude et à son développement. La langue officielle de la République est le russe, mais l'établissement d'une autre langue officielle est possible sur la base d'une volonté populaire exprimée directement dans le cadre d'un référendum (art. 11). La République de Carélie met en œuvre des mesures pour le renouveau, la perpétuation et le libre développement des Caréliens, des Vepses et des Finnois (art.21), établit les composantes régionales et nationales des normes éducatives d'État (art.29). Le droit d'initiative législative est attribué aux organisations non gouvernementales de la République (art. 42). Le gouvernement de la République est mandaté pour assurer la conservation et le développement de la diversité ethnique et culturelle des peuples habitant la Carélie, leur langue et leur culture, pour protéger les droits des minorités nationales, assurer l'entente entre les nationalités et entre les confessions (art. 57). Des municipalités sur des bases nationales peuvent être constituées dans la République (art. 10).

Sur la base des dispositions constitutionnelles ci-dessus mentionnées, la République de Carélie :

- a adopté la loi du 19 avril 2004, «Sur le soutien des pouvoirs publics à la langue carélienne, au vepse et au finnois en République de Carélie »;

- a formé un ministère chargé des Questions de politique nationale, des Relations avec les organisations sociales, religieuses et avec les médias ;

- a créé le Conseil des représentants des Caréliens, des Vepses et des Finnois auprès du président de la République de Carélie ;

- a élaboré des dispositions gouvernementales pour la mise en œuvre des décisions du Conseil des Caréliens de la République (voir, par exemple, les ordonnances du gouvernement de la République de Carélie du 5 août 2014, nº 476 r-P et du 27 février 2010, n 57 r-P) ;

- a intégré les questions du développement ethnosocial et ethnoculturel des Caréliens, des Finnois et des Vepses dans le programme de développement social et économique de la République de Carélie jusqu'à l'année 2015 (approuvé par une loi de la République du 17 octobre 2010) et comme sous-programme «Conservation de l'unité des peuples et des communautés ethniques de Carélie» pour les années 2014-2020 («Karjala est notre maison »; décret du gouvernement de la République de Carélie du 19 décembre 2013, $\left.\mathrm{n}^{\circ} 365-\mathrm{P}\right)$;

- a proclamé l'année 2013 comme année de la langue carélienne et de la culture nationale (ordonnance du président de la République de Carélie du 27 juin 2013, n 191-r).

13 La République des Komis. La population totale est de 901000 habitants, dont 556000 Russes (61,7\%) et 202000 Komis (22,5\%). Par ailleurs, $87 \%$ de l'ensemble des Komis de la Fédération de Russie vivent dans la République.

14 La Constitution de la République dispose que la formation de la République des Komis et que sa dénomination sont liées à l'habitation ancestrale sur son territoire du peuple komi. Celui-ci se voit garantir la conservation et le développement de sa langue, de sa culture traditionnelle et de son mode de vie (art.3). Le komi, au même titre que le 
russe, est reconnu comme langue officielle de la République (art.67). Le mouvement social interrégional "Komi Vojtyr " se voit accorder le droit d'initiative législative (art. 75).

15 Ces dispositions sont complétées et développées par :

- la loi de la République du 28 mai 1992, «Sur les langues officielles de la République des Komis»;

- la loi de la République du 18 novembre 2009, «Sur certaines questions relatives aux arts populaires sur le territoire de la République des Komis »;

- la loi de la République du 1 ${ }^{\text {er }}$ mars 2011, «Sur l'élevage du renne dans la République des Komis ».

16 Pendant dix ans, la loi du 26 mai 1992 « Sur le statut du Congrès du peuple komi » a été appliquée dans la République. Sur saisine du procureur (ce dernier soutenait que l'adoption d'une telle loi relève de la compétence de la Fédération de Russie et qu'elle établit une préférence sans fondement en faveur du peuple komi), la Cour suprême de la République l'a déclarée contraire à la législation fédérale et l'invalide, de sorte qu'elle ne peut pas être mise en œuvre (décision du 17 juillet 2002, $\left.n^{0} 3-30-2002\right)^{16}$. Le législateur de la République l'a ultérieurement abrogée (loi du 12 mai 2003). Néanmoins, l'institution du Congrès des peuples komis continue à exister en fait ${ }^{17}$ et dispose actuellement du soutien des pouvoirs publics (voir, par exemple, ordonnance du gouvernement de la République des Komis du 17 juin 2014, nº 256-r).

Un ministère chargé de la Politique nationale existe dans la République.

La République du Mari El. La population totale est de 696000 habitants, dont 314000 Russes (46\%) et 291000 Maris (41,8\%). Par ailleurs, $52 \%$ de l'ensemble des Maris de la Fédération de Russie vivent dans la République.

La Constitution de la République reconnaît que les appellations «République du Mari El » et « Mari El » sont synonymes (art. ${ }^{\mathrm{er}}$ ) ; le mari (des collines et des plaines) et le russe sont les langues officielles de la République (art. 15).

La République a adopté la loi du 26 octobre 1996, «Sur les langues dans la République du Mari El », met en œuvre un programme d'État «Politique nationale d'État de la République du Mari El pour les années 2013-2020» (approuvée par le décret du gouvernement de la République du 8 octobre 2012, n 387 ), a créé un ministère de la Culture, de la Presse et des Questions des nationalités, dispose d'un centre républicain de la culture marie, encourage la tenue des congrès du peuple mari (voir, par exemple, l'ordonnance du président de la République du 29 décembre 2007, n 329-rp).

21 La République de Mordovie. La population totale est de 835000 habitants, dont 444000 Russes (53,4\%) et 333000 Mordves (40\%). Par ailleurs, $34 \%$ de l'ensemble des Mordves de la Fédération de Russie vivent dans la République.

Conformément à la Constitution de la République, les appellations République de Mordovie et Mordovie sont synonymes (art. $1^{\mathrm{er}}$ ) ; les langues officielles de la République sont le russe et le mordve (mokcha, erza; art.12); le Congrès du peuple mordve dispose du droit d'initiative législative (art. 85).

La République a adopté la loi du 6 mai 1998 «À propos des langues officielles de la République de Mordovie » et a créé les conditions pour l'étude et l'enseignement du russe et du mordve dans les établissements d'enseignement (voir art. 3 de la loi du 8 août 2013, "Sur l'éducation dans la République de Mordovie»); elle garantit la possibilité de s'exprimer en langue mordve au sein du Parlement de la République 
(art. 16 du règlement de l'Assemblée nationale de la République de Mordovie); elle soutient les autonomies culturelles et nationales des Mordves situés dans d'autres sujets de la Fédération de Russie (loi du $1^{\text {er }}$ décembre 2004, «Sur le soutien de l'État aux autonomies nationales et culturelles ») ; elle encourage le développement des métiers d'art et d'artisanat populaires (loi du 14 juin 2000, «Sur les métiers d'art et d'artisanat populaires dans la République de Mordovie»); elle protège les objets du patrimoine culturel du peuple de la République (loi du 12 novembre 2001, «Sur le soutien de l'État dans le domaine culturel »); elle encourage la tenue des congrès du peuple mordve (voir décret du président de la République de Mordovie du 7 avril 2014, n ${ }^{\circ} 83-U G$ ) ; elle a entériné le programme "Harmonisation des relations entre les nationalités et entre les religions dans la République de Mordovie " pour les années 2014-2020 (décret du gouvernement de la République du 18 novembre 2013, n 507) ; elle a créé un ministère chargé de la Politique nationale.

La République d'Oudmourtie. La population totale est de 1517000 habitants, dont 913000 Russes (60\%) et 411000 Oudmourtes (27\%). Par ailleurs, $72 \%$ de l'ensemble des Oudmourtes de la Fédération de Russie vivent dans la République.

La Constitution de la République contient des dispositions sur la nation et le peuple oudmourtes, garantit à ce peuple le maintien de sa langue et de sa culture, manifeste une attention à la diaspora oudmourte qui réside massivement sur le territoire d'autres sujets de la Fédération de Russie, reconnaît les appellations "République d'Oudmourtie » et « Oudmourtie » comme équivalentes (art. $1^{\text {er }}$ ). Les langues officielles de la République sont le russe et l'oudmourte (art. 8). Les biens oudmourtes qui présentent une grande importance pour la conservation de la culture matérielle et spirituelle ne peuvent être aliénés qu'avec l'accord du Conseil d'État de la République (le Parlement; art. 26). Le droit d'initiative législative est reconnu aux organisations sociales par le biais de leurs organes républicains ainsi qu'à un groupe de citoyens comprenant au moins 10000 personnes (art. 37).

La République a adopté, le 6 décembre 2001, une loi «Sur les langues officielles de la République d'Oudmourtie et les autres langues des peuples de la République d'Oudmourtie »; elle prévoit l'enseignement et l'étude de la langue oudmourte dans le système éducatif, une coopération dans l'étude de la langue oudmourte pour les personnes résidant en dehors de la République (art. 5 et 6 de la loi du 21 mars 2014, "Sur la réalisation des compétences en matière d'éducation"); elle garantit la protection des objets du patrimoine culturel (loi du 6 mai 2009, «Sur la réglementation des relations en matière de conservation, d'utilisation, de promotion et de protection par l'État des biens du patrimoine culturel [les monuments historiques et culturels] dans la République d'Oudmourtie ») ; elle a créé les conditions du développement de l'artisanat (décret du gouvernement de la République du 10 novembre 2003, n²69); elle met en œuvre l'aide de l'État pour les congrès organisés par l'association interrégionale «Association de tous les Oudmourtes Udmurt keneš » (voir, par exemple, l'ordonnance du gouvernement de la République du 19 novembre 2012, $\mathrm{n}^{\circ}$ 944-r); elle a approuvé le programme d'État «Développement ethnosocial et harmonisation des relations interethniques pour les années 2013-2015» (décret du gouvernement de la République du 19 août $2013, n^{\circ} 372$ ) ; elle a créé un ministère chargé de la Politique nationale. 
27 Ainsi, cette analyse permet de tirer plusieurs conclusions concernant le statut constitutionnel des peuples finno-ougriens qui ont donné leurs noms à une République éponyme, sujet de la Fédération de Russie :

- la république apparait comme la forme politique et juridique de l'autodétermination d'un peuple. Au fil des décennies, la structure d'État ainsi établie constitue un facteur essentiel et efficace pour le développement des groupes ethniques (Konjuhov, 2009, p. 72). En conséquence, la quête de nouvelles formes de coopération entre le pouvoir et la population ne doit pas aller dans le sens d'un affaiblissement ou d'un rejet de cette organisation politique ${ }^{18}$, mais au contraire tendre à son perfectionnement ;

- un peuple finno-ougrien dans une République donnée ne se distingue pas toujours dans le cadre plurinational des peuples du sujet de la Fédération de Russie. Mais, indépendamment de son effectif, ce peuple, en raison de telles ou telles caractéristiques, se voit reconnaître la qualité de peuple titulaire, ce qui détermine l'organisation politique et l'appellation du sujet de la Fédération de Russie ;

- les peuples finno-ougriens ne sont pas désignés comme des peuples autochtones. Au sens de la Constitution de la Fédération de Russie (art. 69) et de la loi fédérale « Sur les garanties des droits des peuples autochtones minoritaires de la Fédération de Russie », les actes du droit international sur les peuples autochtones ne leur sont pas applicables;

- les Républiques, dans les limites de leurs compétences, protègent les droits linguistiques et culturels des peuples titulaires en adoptant des lois spéciales et des programmes d'État, en créant des ministères chargés des Questions nationales. À cet égard, on peut constater des approches stéréotypées en raison du cadre de la législation fédérale, ainsi que le manque de décisions originales et indépendantes, du fait de la centralisation du pouvoir politique ;

- les Républiques ne garantissent pas juridiquement la représentation du peuple titulaire au sein des organes législatifs et des autres instances du pouvoir d'État. Mais, sous forme de compensation, elles attribuent aux groupements de ces peuples le droit d'initiative législative au parlement régional et soutiennent leur activité. Pour autant, la situation juridique des organes et des associations qui agissent au nom du peuple est incomplète. Ainsi, les conditions dans lesquelles ils sont compétents pour assurer une représentation ethnique ne sont pas claires. Il en est de même pour la procédure visant à leur reconnaissance officielle comme groupements autorisés, ainsi que pour le cadre juridique et les formes de la coopération des associations ethniques avec les organes des pouvoirs publics.

\section{Le statut constitutionnel des peuples finno-ougriens qui font partie des « petits " peuples autochtones}

1. Aux termes de la loi fédérale «Sur les garanties des "petits" peuples autochtones de la Fédération de Russie», les peuples autochtones minoritaires sont les peuples qui vivent sur les territoires du peuplement traditionnel de leurs ancêtres, qui conservent un mode de vie, des activités économiques traditionnels et de subsistance, qui sont moins de 50000 personnes dans la Fédération de Russie, et qui se considèrent comme des communautés ethniques autonomes. C'est bien à ces peuples que s'appliquent la Convention $n^{\circ} 169$ de l'Organisation mondiale du travail «Sur les peuples autochtones et les peuples tribaux dans les États indépendants » (adoptée le 7 juin 1989, et qui n'a pas été ratifiée par la Fédération de Russie) et la Déclaration de l'ONU sur les droits des peuples autochtones (adoptée le 13 septembre 2007). 
29 La Fédération de Russie, en reconnaissant l'originalité de ces communautés ethniques et la nécessaire protection de leur habitat et de leur mode de vie (art. 69, 72, part. 1. m de la Constitution de la Fédération de Russie), leur garantit, par cette loi fédérale, le droit à l'usage de la terre, à l'exploitation traditionnelle des ressources naturelles, à la cogestion, à l'autoorganisation par le biais d'institutions coutumières, à une indemnisation, à la préservation et au développement d'une culture originale, à la protection judiciaire en tenant compte des habitudes et des traditions populaires. Ces dispositions sont précisées dans une série de lois fédérales sur les langues, la culture, l'éducation, l'artisanat (voir ci-dessus), sur la terre ${ }^{19}$, les territoires d'exploitation traditionnelle des ressources naturelles ${ }^{20}$, sur les communautés ${ }^{21}$, sur la faune $e^{22}$, sur la pêche ${ }^{23}$ et la chasse ${ }^{24}$.

2. Des dispositions complémentaires de protection juridique des peuples autochtones minoritaires, parmi lesquels se trouvent des peuples finno-ougriens, sont adoptées par les sujets de la Fédération de Russie.

31 L'okrug autonome des Khantys-Mansis-Ougrie. Il est défini comme le lieu de peuplement originel des «petits » peuples autochtones Khantys et Mansis (art. $1^{\mathrm{er}} \mathrm{du}$ statut de l'okrug). Sur son territoire, on dénombre 19000 Khantys (soit 1,2\% de la population de l'okrug) et 11000 Mansis (soit $0,7 \%$ de la population de l'okrug). Par ailleurs, $92 \%$ de l'ensemble des Mansis de la Fédération de Russie, et environ $60 \%$ de l'ensemble des Khantys de la Fédération de Russie vivent dans l'okrug autonome des Khantys-Mansis-Ougrie.

Le statut de l'okrug autonome garantit à ces peuples la protection par l'État de leurs droits (art. 62), la prise en compte de leurs intérêts dans le cadre de l'utilisation des terres et des autres ressources naturelles (art. 64), ainsi que la possibilité de participer aux affaires publiques grâce à l'Assemblée des représentants des "petits " peuples autochtones du Nord au sein de la Douma (Parlement) de l'okrug autonome (art. 23, 27). La législation de l'okrug, en développant ces dispositions, prévoit des mesures destinées à la préservation et au développement des langues des peuples Khantys et Mansis, au soutien de leur folklore et de leurs médias, de leur artisanat, des communautés, des activités économiques traditionnelles ainsi que des mesures destinées à l'amélioration des rapports de ces peuples avec ceux qui exploitent les ressources du sous-sol.

L'oblast' de Murmansk. Le statut de l'oblast' qualifie les Sames vivant sur son territoire de peuple autochtone de la péninsule de Kola (art.21, par.2). Ils sont plus de 1600 personnes (soit $0,2 \%$ de la population de l'oblast'); près de $90 \%$ des Sames de Russie vivent dans l'oblast' de Murmansk.

D'après le statut de l'oblast', les organes du pouvoir sont compétents pour assister la population same dans la mise en œuvre de ses droits au maintien et au développement de sa langue maternelle, de sa culture nationale, de ses traditions et de ses coutumes. Dans leurs zones de peuplement traditionnel, les Sames ont le droit d'utiliser les formes traditionnelles d'exploitation des ressources naturelles et de la pêche (art. 21). L'oblast' a adopté des lois relatives à l'élevage du renne (du 14 janvier 2003) et à l'aide de l'État en faveur des "petits "peuples autochtones du Nord qui exercent des formes d'activité économique traditionnelle ( $\mathrm{du} 30$ juin 2008). La création des conditions destinées à répondre aux besoins des peuples du Nord pour l'étude de leur langue et de leur littérature relève de la compétence du gouvernement en vertu de la loi du 28 novembre 2013. Un Conseil des représentants des peuples autochtones minoritaires du Nord a été créé auprès du gouvernement de l'oblast' pour protéger les droits et les 
intérêts juridiques des Sames (décret du gouvernement de l'oblast' de Murmansk du 11 février 2009, $\mathrm{n}^{\circ}$ 57-PP).

La République de Carélie. La Constitution de la République mentionne les Vepses, sans les qualifier de " petit " peuple autochtone du Nord, au même titre que les Caréliens et les Finnois (art. 21). Dans la République, les Vepses sont près de 3500 (soit 0,53 \% de la population de la République); cela représente près de $60 \%$ de leur effectif en Fédération de Russie.

La Constitution de la Carélie garantit la mise en œuvre de dispositions pour le renouveau, la préservation et le libre développement des Vepses (art. 21). La loi garantit le soutien de l'État à la langue vepse. Les Vepses disposent d'une représentation au sein du Conseil des représentants des Caréliens, des Vepses et des Finnois de la République de Carélie auprès du président de la République (ordonnance du président de la République de Carélie du 6 mars 2014, n 75-r).

L'oblast' de Pskov. Les Setus (Setos ${ }^{25}$ ) vivent sur le territoire de l'oblast'. Ils sont 123 personnes (soit $0,02 \%$ de la population de l'oblast'). Ce peuple fait partie des " petits » peuples autochtones. Dans le statut de l'oblast', il est identifié comme l'ethnie seto, à laquelle sont conférés des droits sur l'habitat ancestral, pour la préservation de sa spécificité, de sa langue, de ses coutumes et de ses traditions, ainsi qu'un droit à l'autoadministration (art. 21). Les dispositions destinées à protéger les Setus (Setos) consistent principalement dans l'allocation de ressources budgétaires de l'oblast' pour le soutien aux associations de ce peuple, pour la participation de ses représentants aux conférences, séminaires et forums consacrés aux problèmes de préservation des peuples minoritaires, pour l'aide sociale aux familles setus qui ont des enfants, etc.

C'est en République d'Oudmourtie que vivent les Besermans. Ils sont plus de 2000 (soit $0,14 \%$ de la population de la République); $96 \%$ des Besermans vivent en Oudmourtie.

Les Besermans, qui ont le statut de " petit " peuple autochtone minoritaire, ne sont pas mentionnés dans la Constitution de la République et ne disposent pas spécifiquement de droits (ce qui, évidemment, ne remet pas en cause les droits reconnus par la législation fédérale en faveur des peuples de ce groupe). Par deux décisions du présidium du Soviet suprême de la République d'Oudmourtie du 2 juillet 1992, $\mathrm{n}^{\circ} 735$-XII, "Sur le rétablissement du nom historique du peuple beserman » et du Conseil des ministres de la République d'Oudmourtie du 29 juillet $1992, n^{\circ} 293$, ce peuple a été reconnu avec cette dénomination comme nationalité autonome. Des dispositions ont également été adoptées pour l'auto-identification ethnique des Besermans, la publication d'un dictionnaire de la langue besermane, de manuels scolaires et d'un recueil de musique et de chants. En tant que groupe ethnique particulier, les Besermans sont mentionnés dans la Conception de la politique nationale d'État de la République d'Oudmourtie (approuvée par un décret du Conseil d'État de la République du 6 février 1998, n 584-I) et dans le programme d'État de la République d'Oudmourtie «Développement ethnosocial et harmonisation des relations interethniques pour la période 2013-2015 " (approuvé par un décret du gouvernement de la République d'Oudmourtie du 19 août 2013, n³ 372).

Dans l'oblast' de Leningrad vivent les peuples votes ( 33 personnes) et ingriens (169 personnes). Ils ont le statut de "petits » peuples autochtones, mais leur situation n'apparaît pas dans le statut de l'oblast'. Cependant, la Conception du développement social et économique de l'oblast' de Leningrad jusqu'en 2025 (approuvée par la loi de 
l'oblast' du 28 juin 2013) reconnaît que les Votes et les Ingriens constituent une population autochtone ancienne du nord-ouest de la Russie. Ils sont concernés par le «Sous-programme de soutien à la spécificité ethnoculturelle des peuples autochtones minoritaires vivant sur le territoire de l'oblast' de Leningrad pour les années 2013-2016 ", qui constitue une partie du programme d'État " Développement social durable dans l'oblast' de Leningrad» (approuvé par une décision du gouvernement de l'oblast' le 14 novembre 2013, n 399), s'applique à ces peuples.

3. Cette étude montre que la législation russe sur les " petits " peuples autochtones vise dans une large mesure à protéger les droits des peuples autochtones minoritaires du Nord, de Sibérie et d'Extrême-Orient. Cela est attesté par le contenu et la thématique des lois fédérales et régionales adoptées dans ce domaine. Les dispositions de cette législation s'appliquent difficilement aux autres peuples qui font partie des " petits » peuples autochtones. Dès lors, ils demeurent des peuples «non septentrionaux » qui ne revendiquent pas et il n'y a pas d'activité législative qui se développe à leur égard.

Si on veut décrire la législation contemporaine applicable aux "petits » peuples autochtones minoritaires, notamment aux peuples du Nord, de Sibérie et d'Extrême-Orient, on peut constater, dans l'ensemble, un état de stagnation et observer une négation des situations juridiques anciennes ${ }^{26}$. Pour la période postérieure à l'année 2000, de nombreux actes juridiques officiellement prévus et attendus par les communautés des peuples autochtones minoritaires ${ }^{27}$ (par exemple, sur la procédure d'attribution de la qualité de citoyen de la Fédération de Russie aux peuples autochtones minoritaires du Nord, de Sibérie et d'Extrême-Orient; sur la procédure d'implantation des zones d'élevage du renne sur la base d'une utilisation permanente perpétuelle - ainsi que sur la procédure de localisation de territoires destinés à l'usage de la chasse par les membres des "petits » peuples autochtones du Nord, de Sibérie et d'Extrême-Orient; sur le mécanisme de conciliation et de prise de décision dans les relations réciproques des peuples autochtones minoritaires du Nord avec les acteurs économiques; sur le projet expérimental de création de territoires d'exploitation traditionnelle des ressources naturelles des peuples autochtones minoritaires; sur l'accès prioritaire des peuples autochtones minoritaires du Nord aux zones de chasse et au gibier et, d'une manière générale, aux ressources naturelles renouvelables) ne sont pas devenus une réalité. Cela veut dire que l'on peut constater une parodie des intentions juridiques en ce qui concerne la gestion des rapports avec les peuples « autochtones ».

Lors de la dernière décennie, les modifications de la législation " autochtone " se sont principalement manifestées par la suppression de dispositions garantissant les droits des peuples autochtones minoritaires ${ }^{28}$. En particulier, actuellement, à la différence du passé, ne sont pas prévus :

- la possibilité, pour les membres de ces peuples, d'obtenir une propriété héréditaire viagère sur les terres ainsi que leur usage gratuit ;

- le classement des territoires d'exploitation traditionnelle des ressources naturelles des "petits» peuples autochtones du Nord, de Sibérie et d'Extrême-Orient comme territoires naturels bénéficiant d'une protection particulière ; actuellement, ils sont seulement qualifiés de «territoires particulièrement protégés» et, en conséquence, les limitations à la commercialisation des terres ne leur sont pas applicables, et les projets d'exploitation économique sur ces territoires ne relèvent pas de l'expertise écologique du gouvernement ;

- l'octroi de gré à gré de terres pour la pêche et la chasse traditionnelles ; 
- l'obligation d'évaluer la possibilité d'un impact négatif des projets sur le mode de vie traditionnel et l'exploitation des ressources naturelles des peuples autochtones minoritaires ;

- l'utilisation par les sujets de la Fédération de Russie d'une partie des redevances liées à l'utilisation des ressources du sous-sol en faveur du développement social et économique des peuples autochtones minoritaires ;

- l'organisation d'une autoadministration locale, en raison d'un peuplement important des peuples autochtones minoritaires sur un territoire communal, et la possibilité de transférer certaines compétences des pouvoirs locaux aux communautés de ces peuples ;

- la faculté pour les sujets de la Fédération de Russie d'établir des quotas de représentation des peuples autochtones minoritaires dans leurs organes législatifs et représentatifs d'autoadministration locale.

L'étude de la législation et des activités de défense des droits de l'homme laisse apparaître, parmi d'autres éléments, que la Fédération de Russie ne dispose pas d'un système stable de gestion publique des affaires concernant les «petits " peuples autochtones. Par exemple, la question de ces peuples a été transférée à la compétence du ministère de la Culture de la Fédération de Russie par le décret du président de la Fédération de Russie du 8 septembre 2014, $\mathrm{n}^{\circ}$ 612, «Sur la suppression du ministère du Développement régional de la Fédération de Russie ${ }^{29}$ ». Depuis 1993, et l'entrée en vigueur de l'actuelle Constitution, c'est le onzième changement dans leur « rattachement» administratif. Cela, de mon point de vue, démontre les difficultés à appréhender le statut des peuples autochtones minoritaires en Russie et complique la résolution de leurs problèmes.

\section{Le statut constitutionnel des peuples finno-ougriens qui constituent des minorités nationales}

La législation russe ne définit pas les minorités nationales et n'indique pas les communautés qui en font partie. Toutefois, elle reconnaît leur existence en Fédération de Russie par :

- l'attribution à la Fédération de Russie d'une compétence en matière de réglementation et de protection des droits des minorités nationales, ainsi que l'attribution d'une compétence partagée entre la Fédération de Russie et ses sujets pour la protection des droits des minorités nationales (art.71, c; art 72, par.1, b, de la Constitution de la Fédération de Russie);

- la ratification par une loi fédérale du 18 juin 1998 de la Convention-cadre sur la protection des minorités nationales ${ }^{30}$.

En règle générale, les minorités nationales comportent les représentants de groupes ethniques qui vivent sur le territoire d'un État donné et qui en ont la citoyenneté, mais qui n'appartiennent pas à la nation originaire et qui se perçoivent eux-mêmes comme une communauté nationale. Des critères de l'appartenance à un tel groupe peuvent être l'auto-identification en tant que telle, l'origine, des caractéristiques culturelles clairement prononcées (une culture originale, la langue, la religion, les coutumes), l'existence d'une organisation sociale qui permet une coopération à l'intérieur de la minorité ethnique ainsi qu'avec les autres groupes de la population ${ }^{31}$. Selon cette approche peut être considéré comme une minorité nationale au sens large dans la 
Fédération de Russie n'importe quel groupe ethnique différent de la communauté majoritaire que constitue le peuple russe ${ }^{32}$.

Cependant, les peuples qui ont, en Russie, leurs propres structures gouvernementales dans le cadre des Républiques, et les peuples autochtones minoritaires dotés d'un statut particulier, ne se reconnaissent pas comme des minorités nationales. C'est pourquoi, en tenant compte de la spécificité de la situation en Russie, il est exact de classer comme minorités nationales seulement ceux qui ne font pas partie desdits groupes ethniques ${ }^{33}$.

Ainsi sont considérés comme des minorités nationales de facto, au sein des peuples finno-ougriens de la Fédération de Russie :

- les Finnois qui vivent dans la République de Carélie (plus de 8000 personnes), dans l'oblast' de Leningrad (plus de 4000 personnes) et à Saint-Pétersbourg (plus de 2500 personnes) ;

- les Estoniens qui vivent dans le kraj de Krasnojarsk (plus de 2300 personnes), dans l'oblast' d'Omsk (plus de 2000 personnes), à Saint-Pétersbourg (plus de 1500 personnes), dans l'oblast' de Leningrad (environ 800 personnes), dans l'oblast' de Pskov (plus de 600 personnes) et dans plusieurs autres régions de Russie ;

- les Komis permiaks qui vivent dans le kraj de Perm' (plus de 81000 personnes);

- les Caréliens, les Komis, les Maris, les Mordves et les Oudmourtes qui vivent en dehors des frontières de la République dans laquelle ils constituent fondamentalement le peuple autochtone (titulaire).

En application de la loi du 17 juin 1996 «Sur l'autonomie nationale et culturelle » ${ }^{34}$, ces communautés ethniques, qui forment une minorité nationale, ont le droit de créer des autonomies (locales, régionales et fédérales) sur une base nationale et culturelle comme forme d'organisation sociale, en vue du règlement autonome des questions de sauvegarde de leur identité, du développement de la langue, de l'éducation et de la culture nationale. De telles organisations sont dotées de certains droits et peuvent recevoir une aide matérielle et financière des organes du pouvoir d'État ${ }^{35}$.

Le droit de former une autonomie nationale et culturelle est particulièrement demandé par les peuples finno-ougriens qui constituent une minorité nationale. Actuellement, de telles organisations ont été créées par les Finnois (ville de Saint-Pétersbourg, oblast' de Leningrad) ${ }^{36}$, les Estoniens (ville de Krasnojarsk), les Komis permiaks (République des Komis), les Oudmourtes (République du Tatarstan), les Maris (Républiques d'Oudmourtie, du Bachkortostan, du Tatarstan, oblast' de Leningrad), les Mordves (oblast' de Samara, de Penza et d'Uljanovsk, Républiques de Tchouvachie, du Bachkortostan et du Tatarstan), les Caréliens (oblast' de Tver').

51 Le statut de certaines minorités nationales comporte des spécificités. En particulier, en ce qui concerne les Finnois de Russie, il faut tenir compte de la décision du Soviet suprême de la Fédération de la Russie du 29 juin 1993, n 5291-1, « Sur la réhabilitation des Finnois de Russie $»^{37}$, par laquelle ces derniers ont été rétablis dans leurs droits et une aide d'État leur est garantie pour leur réinstallation culturelle et économique sur le territoire de la Fédération de Russie. En outre, la situation constitutionnelle des Finnois dans la République de Carélie (pour mémoire, elle fut la République socialiste soviétique carélo-finnoise au sein l'Union des républiques socialistes soviétiques entre le 31 mars 1940 et le 16 juillet 1956) ne se distingue pratiquement pas de la situation des Caréliens dans cette République. En conséquence, les représentants du peuple finnois, lorsqu'ils réfléchissent d'une façon conceptuelle sur le statut de leur groupe ethnique, utilisent les notions de « diaspora » et de « peuple autochtone $»^{38}$. 

période, leur développement comme groupe ethnique eut lieu dans le cadre d'une formation territoriale (étatique) nationale propre, à savoir un okrug national (autonome ; 1925-2005). L'ancien statut de l'okrug autonome des Komis permiaks, en date du 14 décembre 1994, définissait l'okrug autonome comme le lieu du peuplement compact de la population autochtone des Komis permiaks, qui a donné son nom à l'okrug. Les organes du pouvoir d'État de l'okrug autonome devaient contribuer au développement de la culture et de la langue, mais également créer les conditions pour satisfaire les besoins culturels et nationaux du peuple komi permiak ainsi que des minorités nationales qui vivent sur le territoire de l'okrug (art. 5, par. 4). Ce qui signifie que, d'un point de vue juridique, le peuple komi permiak ne constituait pas une minorité nationale, ce qui correspondait aussi à une situation réelle: lors du recensement de 2002, les Komis permiaks représentaient $59 \%$ de la population de l'okrug autonome. l'oblast' de Perm' ${ }^{39}$, qui aboutit à la formation d'un nouveau sujet de la Fédération de Russie, le kraj de Perm'. L'okrug autonome des Komis permiaks devint alors l'okrug des Komis permiaks, qui constitue une unité administrative et territoriale du kraj de Perm' avec un territoire unique et un statut particulier, déterminé par le statut du kraj conformément à la législation de la Fédération de Russie ${ }^{40}$. Le statut du kraj de Perm' du 27 avril 2007 stipule que le kraj de Perm' est le successeur de l'oblast' de Perm' et de l'okrug autonome des Komis permiaks (art. ${ }^{\mathrm{er}}$, par. 2) ; l'okrug autonome des Komis permiaks devient, au sein du kraj de Perm', l'okrug des Komis permiaks et obtient le statut d'unité administrative et territoriale dans les limites géographiques de l'ancien okrug autonome (art. 6, par. 4; art. 35, par. 1); l'okrug des Komis permiaks n'a pas d'organe représentatif, et le pouvoir exécutif sur son territoire est assuré par le ministère du kraj chargé des affaires de l'okrug des Komis permiaks, dont le ministre est simultanément le responsable de l'administration dudit okrug (art. 37, 40); les organes du pouvoir d'État du kraj de Perm' créent les conditions pour la sauvegarde et le développement de la langue, de la culture spirituelle et des autres composantes de l'identité ethnique du peuple komi permiak. Dans le statut du kraj, les Komis permiaks ne sont pas dénommés comme peuple ou population autochtone ; ils ne sont même pas qualifiés de minorité nationale. Cependant, ils représentent actuellement $3 \%$ de l'ensemble de la population du kraj de Perm', c'est-à-dire qu'ils constituent en réalité dans ce sujet de la Fédération de Russie une minorité nationale.

\section{Conclusion}

La présente étude permet de tirer quelques conclusions générales. Premièrement : les peuples finno-ougriens de Russie, qui possèdent plusieurs caractéristiques culturelles et linguistiques communes, connaissent des situations réelles, des évolutions comme ethnie, des besoins sociaux et culturels, des effectifs, etc., différents. D'une manière objective, cela détermine la diversité des statuts de ces peuples, en tenant compte des normes juridiques internationales et en conformité avec la Constitution de la Fédération de Russie et la législation en vigueur.

Deuxièmement : la Constitution de la Fédération de Russie garantit l'égalité juridique des peuples et leur droit à l'autodétermination dans la Fédération de Russie, l'égalité 
des droits et des libertés individuelles indépendamment de la nationalité et de la langue. Cependant, un statut constitutionnel approprié (en cas de manifestation de volonté positive d'une ethnie et d'une reconnaissance par l'État) permet aux communautés ethniques d'obtenir des moyens supplémentaires pour la protection juridique par l'État de leurs droits, notamment politiques, sociaux et culturels. Troisièmement: les deux dernières décennies ont été marquées par l'aspiration des peuples finno-ougriens à une consolidation. Cela est encouragé, notamment, par les congrès mondiaux et panrusses de ces peuples organisés périodiquement, par l'activité de leurs organisations telles que le Comité consultatif des peuples finno-ougriens et l'Association des peuples finno-ougriens de Russie ${ }^{41}$. Il est manifeste qu'une telle coopération directe des peuples, en vue du règlement de problèmes communs, sera d'autant plus efficace qu'il sera davantage tenu compte de leurs spécificités et de leurs statuts (qui conditionnent le choix de mesures appropriées pour le développement et la protection des ethnies), ainsi que leurs relations avec les organes du pouvoir d'État.

\section{BIBLIOGRAPHIE}

Atlas, 2009 = НАРОДЫ РОССИИ: АТЛАС КУЛЬТУР И РЕЛИГИЙ (Les peuples de Russie, Atlas des cultures et des religions), ОТВ. РЕД. А.В. ЖУРАВСКИЙ, О.Е. КАЗЬМИНА, В.А. ТИШКОВ, 2-Е ИЗД, ИСПР. И ДОП., москвА.

Droit, 2000 = КОНСТИТУЦИОННОЕ ПРАВО. ЭНЦИКЛОПЕДИЧЕСКИЙ СЛОВАРЬ (Droit constitutionnel. Dictionnaire encyclopédique), ОТВ.РЕД. И РУКОВОДИТЕЛЬ АВТОРСКОГО КОЛЛЕКТИВА ПРОФ. С.А. АВАКЬЯН, МОСКВА.

HABRIEVA, 2003 = ХАБРИЕВА ТАЛИЯ ЯРУЛЛОВНА, НАЦИОНАЛЬНО-КУЛЬТУРНАЯ АВТОНОМИЯ В РОССИЙСКОЙ ФЕДЕРАЦИИ (L'autonomie nationale et culturelle dans la Fédération de Russie), москвА.

KOLOMAJNEN, 2010 = КОЛОМАЙНЕН РОБЕРТ ПЕТРОВИЧ, «РОССИЙСКИЕ ФИННЫ: МЕНЬШЕ, ЧЕМ СУБЭТНОС, И БОЛЬШЕ, ЧЕМ ДИАСПОРА » (Les Finnois de Russie ; moins qu'une sous-ethnie et plus qu'une diaspora), РОССИЙСКИЕ ФИННЫ: ВЧЕРА, СЕГОДНЯ, ЗАВТРА, СБОРНИК СТАТЕЙ, ПОСВЯЩЕННЫХ 20-ЛЕТИЮ ИНГЕРМАЛАНДСКОГО СОЮЗА ФИННОВ КАРЕЛИИ (Les Finnois de Russie : hier, aujourd'hui et demain. Recueil des articles consacrés au $20^{\mathrm{e}}$ anniversaire de l'Union ingrienne des Finnois de Carélie), НАУЧ. РЕД. Е.И. КЛЕМЕНТЬЕВ, ПЕТРОЗАВОДСК.

KONJUHOV (ed.), 2008 = КОНюХОВ АЛЕКСЕЙ КИМОВИЧ (ОТВ. РЕД.), ФИННО-УГОРСКИЕ НАРОДЫ РОССИИ: ВЧЕРА, СЕГОДНЯ, ЗАВТРА (Les peuples finno-ougriens de Russie : hier, aujourd'hui et demain), СЫКТЫВКАР.

КRJАŽКОV, 2010 = КРЯЖКОВ ВЛАДИМИР АЛЕКСЕЕВИЧ, КОРЕННЫЕ МАЛОЧИСЛЕННЫЕ НАРОДЫ СЕВЕРА В РОССИЙСКОМ ПРАВЕ (Les peuples autochtones minoritaires du Nord dans le droit russe), МоскВА.

KRJAŽKOV, 2012 = КРЯЖКОВ ВЛАДИМИР АЛЕКСЕЕВИЧ, «РОССИЙСКОЕ ЗАКОНОДАТЕЛЬСТВО О СЕВЕРНЫХ НАРОДАХ И ПРАВОПРИМЕНИТЕЛЬНАЯ ПРАКТИКА: СОСТОЯНИЕ И ПЕРСПЕКТИВЫ» (La 
législation russe concernant les peuples du Nord et son application : état et perspectives), ГОСУДАРСТВО И ПРАВО (État et droit), $n^{\circ}$ 5, cc. 27-35.

Le monde, 1996 = ФИННО-УГОРСКИЙ МИР (СПРАВОЧНИК ПО ИСТОРИИ, КУЛЬТУРЕ И ЯЗЫКУ) (Le monde finno-ougrien. Guide historique, culturel et linguistique), БУДАПЕшт - МоскВА.

Les peuples, 2006 = ФИННО-УГОРСКИЕ И САМОДИЙСКИЕ НАРОДЫ РОССИИ. СТАТИСТИЧЕСКИЙ СБорник (Les peuples finno-ougriens et samoyèdes de Russie. Recueil statistique), СЫктЫВКАР.

MARKOV, 2011 = МАРКОВ ВАЛЕРИЙ ПЕТРОВИЧ, ВОЗРОЖДЕНИЕ В ЭПОХУ ПЕРЕМЕН. СЪЕЗДЫ КОМИ НАРОДА: ДОКУМЕНТЫ И КОММЕНТАРИИ (La renaissance dans une époque de changements. Les congrès du peuple komi : documents et commentaires), сыктывкаР.

OSIPOV, 2004 = ОСИПОВ АЛЕКСАНДР ГЕННАДЬЕВИЧ, НАЦИОНАЛЬНО-КУЛЬТУРНАЯ АВТОНОМИЯ. ИДЕИ, РЕШЕНИЯ, ИНСТИТУТЫ (L'autonomie nationale et culturelle. Idées, décisions, institutions), САНКТ-ПЕТЕРБУРГ.

SIMONOV, 2015 = СИМОНОВ ВЛАДИМИР АЛЕКСАНДРОВИЧ, «О РЕФОРМИРОВАНИИ ФОРМЫ ГОСУДАРСТВЕННОГО ЕДИНСТВА МНОГОНАЦИОНАЛЬНОЙ РОССИИ» (Sur la réforme des modes d'unité de l'État dans la Russie plurinationale) ; СОВЕРШЕНСТВОВАНИЕ МЕСТНОГО САМОУПРАВЛЕНИЯ СКВОзЬ ПРИЗМУ КОНСТИТУЦИоННОЙ эКОНОМИКИ (Amélioration de l'autoadministration locale à travers le prisme de l'économie constitutionnelle), КОЛЛЕКТИВНАЯ МОНОГРАФИЯ ПОД РЕД. ПРОФ. А.Н. костюКовА, Омск.

\section{NOTES}

1. Sur les caractéristiques historiques, démographiques... de ces peuples, voir : Le monde, 1996 ; Les peuples, 2006 ; Atlas, 2009.

2. À ce propos, la Constitution de la République Sahaa (Yakoutie) utilise la notion de "peuple autochtone de la République Sahaa (Yakoutie)» (art. 42). Selon la Constitution de la République de l'Altaj, le territoire de la République constitue la terre ancestrale de l'habitat traditionnel du peuple autochtone (d'après la Constitution, les Altaïens) et des autres peuples (art. 10).

3. En russe : КОРЕННЫЕ МАЛОчИСЛЕННЫЕ НАРОДЫ. C'est là une notion difficile à traduire, car l'adjectif qui qualifie ces peuples autochtones se réfère exclusivement au nombre de leurs ressortissants, qui doit être faible. Mot à mot, ce serait des «peuples numériquement peu nombreux ». Nous choisissons pour la clarté de l'expression l'adjectif " petit », en précisant qu'il ne contient aucune connotation méprisante. En effet, il est important de mentionner la taille de ces peuples car ce statut ne concerne que les peuples de moins de 50000 personnes (N.d.É.).

4. Voir Recueil de la législation de la Fédération de Russie (ci-après Rec. lég. Féd. Rus.), 2000, $\mathrm{n}^{\circ} 14$, p. 1493.

5. Voir Rec. lég. Féd. Rus., 2006, $n^{\circ}$ 17, p. 1905.

6. Voir Rec. lég. Féd. Rus., 1999, nº 18, p. 2208.

7. Attention aux malentendus induits par les pratiques langagières différentes dans les deux pays : il s'agit ici de nationalités au sens ethnique et non politique. Les Finnois et les Estoniens dont il est question sont citoyens de la Fédération de Russie, mais se reconnaissent une ethnicité finnoise ou estonienne, ce qui s'appelle en Russie «nationalité » (N.d.É.).

8. Voir Rec. lég. Féd. Rus., 2012, nº 48, p. 6744.

9. Voir Rec. lég. Féd. Rus., 1999, nº 42, p. 5005.

10. Voir Registre du Congrès des députés du peuple et du Soviet suprême de la République socialiste fédérative soviétique de Russie, 1991, n 50, p. 1740. 
11. Voir Registre du Congrès des députés du peuple et du Soviet suprême de la République socialiste fédérative soviétique de Russie, 1991, nº 46, p. 2615.

12. Voir Rec. lég. Féd. Rus., 1999, nº 2, p. 234.

13. Voir Rec. lég. Féd. Rus., 2002, nº 26, p. 2519.

14. Voir Rec. lég. Féd. Rus., 2012, nº 53, p. 7598.

15. Pour ces données, ainsi que pour les suivantes, on utilise les chiffres de la population totale qui résultent du recensement de 2010 tel que présentés sur Wikipédia (https://ru.wikipedia.org/) [consulté le 28.03.2017].

16. La cour, approuvant la position du procureur, a rendu une décision qui, selon nous, est contestable. En effet, d'une part, lorsqu'un sujet de la Fédération de Russie protège les droits et les libertés des individus et des citoyens ainsi que ceux des minorités nationales, il est juridiquement compétent pour adopter les lois appropriées (art. 72 et 76 de la Constitution de la Fédération de Russie). D'autre part, la Constitution de la Fédération de Russie, en reconnaissant la République des Komis comme sujet de la Fédération de Russie (art. 65), reconnait par là même le rôle spécifique des Komis comme peuple constitutif de ce sujet. En conséquence, la réglementation du fonctionnement des institutions relatives à l'autoorganisation du peuple komi est parfaitement conforme avec son statut au sein de la République, dont la spécificité, rappelons-le encore une fois, est affirmée par la Constitution même de la République des Komis (art. 3).

17. Sur la tenue des congrès du peuple komi et ses décisions, voir Markov, 2011.

18. Cela apparaît, par exemple, dans les discours dans lesquels, sans raisons suffisantes, il est proposé de supprimer en Fédération de Russie les structures étatiques nationales en estimant qu'elles constituent une menace potentielle comme source de séparatisme et de destruction de l'unité de l'État russe (voir Simonov, 2015).

19. Voir le code foncier de la Fédération de Russie du 25 octobre 2001, Rec. lég. Féd. Rus., 2001, $\mathrm{n}^{\circ} 44$, p. 4347.

20. Voir la loi fédérale du 7 mai 2001, "Sur les territoires d'exploitation traditionnelle des ressources naturelles des peuples autochtones minoritaires du Nord, de Sibérie et d'ExtrêmeOrient de la Fédération de Russie », Rec. lég. Féd. Rus., 2001, n² 20, p. 1972.

21. Voir la loi fédérale du 20 juillet 2000 , «Sur les principes généraux d'organisation des communautés des peuples autochtones minoritaires du Nord, de Sibérie et d'Extrême-Orient de la Fédération de Russie, Rec. lég. Féd. Rus., 2000, nº 30, p. 3122.

22. Voir la loi fédérale du 24 avril 1995, «Sur la faune », Rec. lég. Féd. Rus., 1995, nº 17, p. 1462.

23. Voir la loi fédérale du 20 décembre 2004, "Sur la pêche et la conservation des ressources biologiques aquatiques ", Rec. lég. Féd. Rus., 2004, n 52 (partie 1), p. 5270.

24. Voir la loi fédérale du 24 juillet 2009, «Sur la chasse, la conservation des ressources de chasse et l'introduction d'amendements à certains actes législatifs de la Fédération de Russie », Rec. lég. Féd. Rus., 2009, n 30, p. 3735.

25. Le terme «setu» provient de la langue littéraire estonienne. "Seto» est leur autoethnonyme (N.d.R.).

26. Pour une analyse détaillée de la situation, voir Krjažkov, 2012.

27. Voir, par exemple, les ordonnances du gouvernement de la Fédération de Russie du 21 février 2005, no 185-r et du 28 août 2009, nº 1245-r, Rec. lég. Féd. Rus., 2005, nº 9, p 736 ; 2009, $n^{\circ} 36$, p. 4364.

28. Une exception à cette tendance se trouve dans la réglementation de la question de l'enregistrement des membres des peuples autochtones minoritaires ayant un mode de vie nomade et (ou) semi-nomade (voir la loi fédérale du 6 décembre 2011, n 399-LF, Rec. lég. Féd. Rus., 2011, n 50, p. 7341), et la modernisation du code foncier de la Fédération de Russie pour les droits des peuples autochtones minoritaires à l'utilisation des terres (voir la loi fédérale du 
23 juin 2014, nº 171-LF et la loi fédérale du 21 juillet 2014, n 217-LF, Rec. lég. Féd. Rus., 2014, nº 26

[partie 1], p. $3377 ; n^{\circ} 30$ [partie 1], p. 4218).

29. Voir Rec. lég. Féd. Rus., 2014, nº 37, p. 4934.

30. Voir Rec. lég. Féd. Rus., 1998, nº 25, p. 2833.

31. Voir article «Minorité nationale», Wikipédia (https://ru.wikipedia.org/) [consulté le 28.03.2017].

32. Voir, par exemple, le comité consultatif pour l'application de la Convention de Rome sur la protection des minorités nationales. Avis sur la Fédération de Russie, Strasbourg, 13 septembre 2012.

33. On peut noter qu'il a été proposé d'inclure cela dans un des projets de loi fédérale sur les minorités nationales en Fédération de Russie (voir Droit constitutionnel, 2000, p. 376).

34. Voir Rec. leg. Féd. Rus., 1996, nº 25, p. 2965.

35. Voir Habrieva, 2003 et Osipov, 2004.

36. En complément, on peut noter que, dans la République de Carélie, il existe une organisation sociale régionale, « l'Union ingrienne des Finnois de Carélie ».

37. Voir Registre du Congrès des députés du peuple et du Soviet suprême de la République socialiste fédérative soviétique de Russie, 1993, n² 22, p. 1119.

38. Voir Kolomajnen, 2010, p. 10.

39. D'un point de vue formel, elle s'effectua à la suite de référendums qui ont eu lieu au sein des sujets de la Fédération de Russie qui allaient fusionner, mais l'opinion des Komis permiaks sur cette question ne s'est pas spécialement manifestée (à propos d'unifications semblables, voir Kriazhkov, 2010, p. 319-326).

40. Voir la loi constitutionnelle fédérale du 25 mars 2004, «Sur la formation à l'intérieur de la Fédération de Russie d'un nouveau sujet de la Fédération de Russie suite à l'unification de l'oblast' de Perm' et de l'okrug autonome des Komis permiaks» (art. 4, par. 2), Rec. lég. Féd. Rus. 2004, $\mathrm{n}^{\circ} 13, \mathrm{p} .1110$.

41. Voir Les peuples..., 2006, p. 85.

\section{RÉSUMÉS}

L'article analyse les statuts constitutionnels des peuples finno-ougriens en Russie. Il expose les particularités de la situation de ces peuples dans les Républiques, ainsi que celles des peuples autochtones minoritaires et enfin celles des peuples formant une minorité nationale. Il propose également des mesures pour améliorer leur protection juridique.

This article analyses the constitutional statuses of Finno-Ugric peoples in Russia. It exposes the particular features of these peoples in the republics, of the minority native peoples as well as those who form an ethnic minority. It also proposes ways to strengthen their legal protection.

В СТАТЬЕ АНАЛИЗИРУЮТСЯ КОНСТИТУЦИОННО-ПРАВОВЫЕ СТАТУСЫ ФИННО-УГОРСКИХ НАРОДОВ В РОССИИ. ВЫЯВЛЯЮТСЯ ОСОБЕННОСТИ ПОЛОЖЕНИЯ ДАННЫХ НАРОДОВ РЕСПУБЛИК, КОРЕННЫХ МАЛОЧИСЛЕННЫХ НАРОДОВ И НАРОДОВ, НАХОДЯЩИХСЯ В СИТУАЦИИ НАЦИОНАЛЬНОГОМЕНЬШИНСТВА, А ТАКЖЕ ПРЕДЛАГАЮТСЯ МЕРЫ ПО СОВЕРШЕНСТВОВАНИЮ ИХ ПРАВОВОЙ ЗАщИТЫ. 


\section{INDEX}

Mots-clés : minorités nationales, minorités nationales, peuples finno-ougriens, peuples finno-ougriens, peuples autochtones minoritaires, peuples autochtones minoritaires, statut constitutionnel, statut constitutionnel

Keywords : constitutional status, Finno-Ugric peoples, indigenous minorities, national minority Thèmes : droit, droit constitutionnel nomsmotscles Altaïens, Bessermans, Caréliens, Estoniens, Finnois, Ingriens, Khantys, Komis, Komis permiaks, Mansis, Maris, Mordves, Oudmourtes, peuples finno-ougriens, Sames, Setos, Vepses, Votes

motscleset rahvusvähemused, soome-ugri rahvad, põlisrahvastest vähemused, põhiseaduslik staatus

motsclesru КОНСТИТУЦИОННО-ПРАВОВОЙ СТАТУС, КОРЕННЫЕ МАЛОЧИСЛЕННЫЕ НАРОДЫ, НАЦИОНАЛЬНЫЕ МЕНЬШИНСТВА, ФИННО-УГОРСКИЕ НАРОДЫ

Index géographique : Bachkortostan, Carélie, Khantys-Mansis (district autonome), Komi, Krasnojarsk, Leningrad (oblast'), Marij El, Mordovie, Murmansk (oblast'), Oudmourtie, Penza (oblast'), Perm' (kraj) Pskov, Russie, Saint-Pétersbourg, Samara (oblast'), Sibérie, Tatarstan, Tchouvachie, Tver', Uljanovsk (oblast')

disciplines carélien, erza, komi, mari, mari des collines, mari des plaines, mokcha, mordve, oudmourte, russe

Index chronologique : XXIe siècle (début) 